\title{
HARNACK INEQUALITIES FOR FUNCTIONAL SDES DRIVEN BY SUBORDINATE MULTIFRACTIONAL BROWNIAN MOTION
}

\author{
ZHI LI, LITAN YAN AND LIPING XU*
}

Abstract. Being base on the Girsanov theorem for multifractional Brownian motion, which can be constructed by the multifractional derivative operator, we establish the Harnack inequalities for a class of stochastic functional differential equations driven by subordinate multifractional Brownian motion by an approximation technique.

Mathematics subject classification (2020): 60H15, 60G15, 60H05.

Keywords and phrases: Harnack inequality, multifractioanl Brownian motion, subordinator, coupling.

\section{REFERENCES}

[1] A. Ayache, S. Cohen AND J. L. Vehel, The covariance structure of multifractional Brownian motion, with application to long range dependence, Acoustics, Speech, and Signal Processing., 10.1109/ICASSP.2000.860233.

[2] J. H. BAO, F.Y. WANG AND C. G. YUAN, Bismut formulae and applications for functional SPDEs, Bull. Sci. Math., 137, (2013), 509-522.

[3] J. H. BAO, F. Y. WANG AND C. G. YUAN, Asymptotic Log-Harnack inequality and applications for stochastic systems of infinite memory, Stochastic Processes and their Applications., 129, 11 (2019), 4576-4596.

[4] R. F. BAss And D. A. Levin, Harnack inequalities for jump processes, Potential Analysis., 17, (2002), 375-388.

[5] A. Benassi, S. JAFFARD And D. Roux, Elliptic gaussian random processes, Revista Matematica Iberoamericana., 13, 1, (1997), 19-90.

[6] S. BOUdRAHEM AND P. R. Rougier, Relation between postural control assessment with eyes open and centre of pressure visual feed back effects in healthy individuals, Exp. Brain Res., 195, (2009), $145-152$.

[7] B. Boufoussi, M. Dozzi And R. Guerbaz, Sample path properties of the local time of multifractional Brownian motion, Bernoulli., 13, 3, (2007), 849-867.

[8] F. COMte And E. Renault, Long memory continuous time models, J. Econometrics., 73, 1, (1996), $101-149$.

[9] I. CsiszÁr And J. KöRne, Information Theory: Coding Theorems for Discrete Memory-less Systems, Academic Press, New York, 1981.

[10] F. de La, A. L. Perez-Samartin, L. Matnez, M. A. Garcia and A. Vera-Lopen, Longrange correlations in rabbit brain neural activity, Ann. Biomed. Eng. February., 34, 2, (2006), 295299.

[11] C. S. DENG AND X. HUANG, Harnack inequalities for functional SDEs driven by subordinate Brownian motions, Potential Analysis., https://doi.org/10.1007/s11118-020-09882-0.

[12] C. S. Deng AND R. Schilling, Harnack inequalities for SDEs driven by time-changed fractional Brownian motions, Electron. J. Probab., 22, (2017), 1-23.

[13] C. S. DENG, Harnack inequalities for SDEs driven by subordinate Brownian motions, J. Math. Anal. Appl., 417, (2014), 970-978.

[14] X. L. FAN, Harnack inequality and derivative formula for SDE driven by fractional Brownian motion, Sci. China Ser. A., 561, (2013), 515-524. 
[15] X. L. FAN, Harnack-type inequalities and applications for SDE driven by fractional Brownian motion, Stoch. Anal. Appl., 32, (2014), 602-618.

[16] F. GONG AND F. Y. WANG, Heat kernel estimates with application to compactness of manifolds, Q. J. Math., 52, (2001), 171-180.

[17] F. A. Harang, T. Nilssen and F. N. Proske, Giranov theorem for multifractional Brownian processes, arXiv:1706.07387v1, (2017).

[18] X. HUANG AND S. Q. ZHANG, Mild solutions and Harnack inequality for functional stochastic partial differential equations with Dini drift, J. Theoret. Probab., 32, (2019), 303-329.

[19] Z. LI, Shift Harnack inequality and integration by parts formula for functional SDEs driven by fractional Brownian motion, Proceedings of the American Mathematical Society., 144, (2016), 26512659.

[20] M. LI, S. C. Lim, B. J. HU AND H. Feng, Towards describing multi-fractality of trafficusing local Hurst function, in: Lecture Notes in Computer Science., 4488, (2007), 1012-1020.

[21] Z. LI AND L. T. YAN, Harnack inequalities for SDEs driven by subordinator fractional Brownian motion, Statistics and Probability Letters., 134, (2018), 45-53.

[22] S. C. Lim, Fractional Brownian motion and multifractional Brownian motion of Rieman-Liouville type, Journal of Physics A General Physics., 34, (2001), 1301-1310.

[23] A. Mimica AND P. KIm, Harnack inequalities for subordinate Brownian motions, Electronic Journal of Probability., 17, (2012), 1-23.

[24] S. V. Muniandy AND S. C. Lim, Modeling of locally self-similar processes using multifractional Brownian motion of Riemann-Liouville type, Phys. Rev. E., 63, 4, (2001), 046104.

[25] M. NIU AND B. XIE, Wang's Harnack inequalities for space-time white noises driven SPDEs with two reflecting walls and their applications, J. Math. Anal. Appl., 469, (2019), 568-593.

[26] R. F. Peltier And J. L. VÉHel, Multifractional Brownian motion: definition and preliminary results, inria-00074045 (1995).

[27] K. V. Ral' Chenko and G. M. Shevchenko, Path properties of multifractal Brownian motion, Theor. Probability and Math. Statist., 80, (2010), 119-130.

[28] M. RAO, R. SONG AND Z. VONDRAČEK, Green function estimates and Harnack inequality for subordinate Brownian motions, Potential Analysis., 25, (2006), 1-27.

[29] M. RÖCKNER AND F. Y. WANG, Harnack and functional inequalities for generalized Mehler semigroups, J. Funct. Anal., 203, (2003), 237-261.

[30] M. RÖCKNER AND F. Y. WANG, Supercontractivity and ultracontractivity for (non-symmetric) diffusion semigroups on manifolds, Forum Math., 15, (2003), 893-921.

[31] M. RÖCKNER AND F. Y. WANG, Log-Harnack inequality for stochastic differential equations in Hilbert spaces and its consequences, Inf. Dim. Anal. Quantum Probab. Rel. Top., 13, (2010), 2737.

[32] M. Rypdal AND K. Rypdal, Testing hypotheses about sun-climate complexity linking, Phys Rev Lett., 104, 12, (2010), 128-151.

[33] S. G. S Амко, Fractional Integration of Variable Order, 1995.

[34] J. ShaO, Harnack inequalities and heat kernel estimates for SDEs with singular drifts, Bull. Sci. Math., 137, (2013), 589-601.

[35] J. ShaO, F. Y. WANG AND C. YuAn, Harnack inequalities for stochastic (functional) differential equations with non-Lipschitzian coefficients, Electron. J. Probab., 17, (2012), 1-18.

[36] I. Simonsen, Measuring anti-correlations in the nordic electricity spot market by wavelets, Physica A., 322, 1, (2003), 597-606.

[37] F. Y. WANG, Logarithmic Sobolev inequalities on noncompact Riemannian manifolds, Probab. Theory Related Fields., 109, (1997), 417-424.

[38] F. Y. WANG, Harnack inequalities for log-Sobolev functions and estimates of log-Sobolev constants, Ann. Probab., 27, (1999), 653-663.

[39] F. Y. WANG AND J. WANG, Harnack inequalities for stochastic equations driven by Lévy noise, J. Math. Anal. Appl., 410, (2014), 513-523.

[40] W. WANG, Harnack inequality, heat kernel bounds and eigenvalue estimates under integral Ricci curvature bounds, Journal of Differential Equations., 269, 2, (2020), 1243-1277.

[41] F. Y. WANG AND Y. C. YUAN, Harnack inequalities for functional SDEs with multiplicative noise and applications, Stochastic Processes and their Applications., 121, 11, (2011), 2692-2710. 
[42] W. WANG AND P. ZHANG, Some gradient estimates and Harnack inequalities for nonlinear parabolic equations on riemannian manifolds, Mathematische Nachrichten., 290, 11-12, (2017), 1905-1917.

[43] L. WANG AND X. ZHANG, Harnack inequalities for SDEs driven by cylindrical $\alpha$-stable processes, Potential Anal., 42, (2015), 657-669.

[44] W. Willinger, W. Leland, M. TAQQU AND D. Wilson, On self-similar nature of ethernet traffic, IEEE/ACM Trans Networking., 2, 1, (1994), 1-15.

[45] L. T. YAN AND X. W. YIN, Harnack inequality and derivative formula for stochastic heat equation with fractional noise, Electron. Comm. Probab., 23, 35, (2018), 1-11.

[46] L. T. YAN AND X. W. Y IN, Bismut formula for a stochastic heat equation with fractional noise, Statist. Probab. Lett., 137, (2018), 165-172.

[47] X. W. Yin, G. J. SHEN AND Z. L. GAO, Harnack inequality for stochastic heat equation driven by fractional noise with Hurst index $H>1 / 2$, Journal of Mathematical Inequalities., 14, 4, (2020), 1113-1122.

[48] X. C. ZHANG, Derivative formulas and gradient estimates for SDEs driven by $\alpha$-stable processes, Stochastic Processes and their Applications., 123, (2013), 1213-1228. 